\title{
Las percepciones de docentes costarricenses de francés sobre la enseñanza remota de emergencia en el año 2020 en la Universidad de Costa Rica
}

Perceptions of French Costa Ricans professors on emergency remote education in 2020 at the University of Costa Rica

\section{Volumen 21, Número 3 \\ Setiembre - Diciembre \\ pp. 1-36}

Ileana Arias Corrales

Kuok-Wa Chao Chao

\section{Citar este documento según modelo APA}

Arias Corrales, Ileana. y Chao Chao, Kuok-Wa. (2021). Las percepciones de docentes costarricenses de francés sobre la enseñanza remota de emergencia en el año 2020 en la Universidad de Costa Rica. Revista Actualidades Investigativas en Educación, 21(3), 1-36. Doi. 10.15517/aie.v21i3.46457 


\title{
Las percepciones de docentes costarricenses de francés sobre la enseñanza remota de emergencia en el año 2020 en la Universidad de Costa Rica
}

\author{
Perceptions of French Costa Ricans professors on emergency remote education in 2020 at \\ the University of Costa Rica
}

\author{
Ileana Arias Corrales ${ }^{1}$ \\ Kuok-Wa Chao Chao ${ }^{2}$
}

\begin{abstract}
Resumen: Durante el I ciclo de 2020 las personas docentes de la Universidad de Costa Rica adaptaron, en pocos días, sus cursos presenciales a la modalidad virtual en un contexto de emergencia sanitaria. Tomando en cuenta este contexto, se propuso una investigación cualitativa de tipo exploratorio en la que se solicitó a un grupo de personas docentes de francés $(N=19)$ responder a un cuestionario en línea en el mes de julio de 2020. El objetivo que motivó esta investigación, fue analizar las percepciones de las personas docentes sobre la virtualidad (ventajas y dificultades). Las percepciones encontradas pertenecen en primer lugar al nivel socioafectivo, seguidas por percepciones sobre aspectos pedagógicos, logísticos y tecnológicos. En cuanto a las ventajas, todas se centran en el estudiantado y sobre todo en el desarrollo de su autonomía y la autogestión del aprendizaje. Las personas docentes apuestan por un modelo de enseñanza en el que haya cursos presenciales, híbridos (presencialidad cursos en línea) y cursos virtuales en lugar de una virtualidad total de los cursos de francés. Se concluyó que esta modalidad de enseñanza impuesta al inicio de la pandemia conlleva grandes cambios de los modelos actuales e implica una mayor capacitación del personal docente para adaptarse y adaptar sus cursos.
\end{abstract}

Palabras clave: COVID-19, francés, enseñanza a distancia, percepción.

Abstract: During the first cycle of 2020, the professors of the University of Costa Rica adapted, in a few days, their face-to-face courses to the virtual modality in a context of health emergency. Taking this context into account, qualitative exploratory research was proposed requesting a group of French professors $(N=19)$ to respond to an online questionnaire in July 2020. The objective that motivated this research was to analyze teachers' perceptions of virtuality (advantages and difficulties). The perceptions found belong first of all to the socio-affective level, followed by perceptions of pedagogical, logistical and technological difficulties. As for the advantages, they all focus on studying and above all on the development of their autonomy and self-management of learning. Teachers opt for a teaching model in which there are face-to-face courses, hybrids (presence - online courses) and virtual courses instead of a total virtuality of French courses. It is concluded that this type of teaching imposed at the beginning of the pandemic entails major changes in current models and involves greater training of teaching staff to adapt and adapt their courses.

Keywords: COVID-19, french, online education, perception.

\footnotetext{
1 Profesora asociada de la Universidad de Costa Rica, en la Escuela de Lenguas Modernas, San José, Costa Rica. Dirección electrónica: Ileana.arias@ucr.ac.cr Orcid https://orcid.org/0000-0002-8107-5068

2 Profesor catedrático de la Universidad de Costa Rica, en la Escuela de Lenguas Modernas, además Director del Instituto Confusio de la misma universidad, San José, Costa Rica. Dirección electrónica: kuok.chao@ucr.ac.cr Orcid https://orcid.org/0000$\underline{0003-0683-1481}$
}

Artículo recibido: 5 de abril, 2021

Enviado a corrección: 25 de junio, 2021

Aprobado: 16 de agosto, 2021 


\section{Introducción}

En su síntesis sobre la educación en tiempos del COVID (2020), la UNESCO señala que la pandemia provocada por el COVID-19 ha suscitado la mayor crisis de los sistemas educativos de la historia. Esta situación acarreó el cierre parcial o total de jardines de infantes, de escuelas, colegios y universidades; o bien, la adopción de una educación virtual improvisada. Ya para abril de 2020, el "94\% del estudiantado mundial sufría el impacto de la pandemia, es decir, 1,58 millares de niños y jóvenes [...] en 200 países" (UNESCO, 2020, p. 5).

Este hecho transformó la manera en la que se concebía, se comprendía y se vivían los procesos educativos. La UNESCO (2020) señala que, en lo que respecta a estudios universitarios, "los cursos pregrabados y las plataformas en línea sustituyeron la enseñanza tradicional, pero algunas universidades suspendieron sus cursos hasta nuevo aviso debido a falta de equipos informáticos para sus estudiantes y docentes" (UNESCO, 2020, p.8). En el contexto de la Universidad de Costa Rica (UCR), a partir de la segunda semana de marzo de 2020, la totalidad de los cursos cambiaron de modalidad presencial a modalidad virtual pese a las dificultades propias de cada Facultad.

Algunas investigaciones como la de Kikut Valverde (2020) y la de Villanueva Paredes, Calcina Málaga, Chipa Pandia, Fuentes Calsina y Suxso Condori (2020) encontraron que áreas como ciencias básicas, educación, arquitectura e ingenierías son percibidas por el estudiantado como áreas cuyos cursos son difíciles de adaptar a la virtualidad dada su naturaleza. Tejedor, Cervi, Tusa y Parola (2020) coinciden con los resultados obtenidos por Kikut Valverde (2020) y Villanueva Paredes et al. (2020), y agregan que el paso de la presencialidad a la virtualidad ha sido un cambio negativo según la percepción del alumnado debido a la mala preparación de la persona docente, al aumento de lecturas y de trabajo para el estudiantado, a la falta de estímulo y a la sensación de aprender menos que en la presencialidad.

En la Escuela de Lenguas Modernas (ELM) existió durante dos décadas el programa de francés a distancia, en el cual la mayoría de los cursos contaba con sesiones presenciales (tutorías) una vez por semana y con trabajo autónomo intercalado. La licenciatura en la enseñanza del francés, creada hace ya una década, siguió este mismo modelo. Los demás cursos del área de francés nunca se habían propuesto de manera virtual ni en un modelo híbrido como fue el caso del programa de Francés a distancia y de la Licenciatura en la enseñanza del francés. Por lo anterior, es necesario recalcar que, en las secciones de Francés 
de primer año, II a IV año de carrera del Bachillerato en Francés y en Francés para otras carreras, hasta el año 2019, el 100\% de los cursos se impartían en modalidad presencial. En consecuencia, se puede afirmar que la virtualidad fue, en el caso del equipo de docentes de francés, una modalidad de enseñanza completamente nueva.

Por lo tanto, esta investigación tiene como objetivo principal documentar las percepciones del personal docente universitario de francés de la UCR sobre la experiencia vivida en sus cursos durante el contexto de la pandemia del COVID-19 en el año 2020. Como objetivos específicos se proponen los siguientes: (1) identificar, por un lado, las ventajas y las dificultades del personal docente universitario de francés durante el contexto de la pandemia del COVID-19 en el año 2020 y, por otro, (2) describir las percepciones del personal docente universitario de francés con respecto a los cursos que se considerarían aptos para la enseñanza remota en un contexto post pandémico.

\subsection{Estado de la cuestión}

En cuanto a las investigaciones realizadas sobre esta temática, se identifican algunas sobre la experiencia docente de diversas disciplinas de la enseñanza a distancia durante la pandemia. En un estudio cuantitativo con 1400 personas docentes de diversos países, Silas Casillas y Vázquez Rodríguez (2020) obtienen como resultados principales que las dificultades son, a menudo, el acceso a las plataformas por parte del estudiantado, la combinación de tareas domésticas y académicas, los problemas de Internet, la falta de condiciones adecuadas en el hogar para realizar el trabajo y las distracciones del entorno. Identifican también que el profesorado experimenta diversas emociones como la confianza, la saturación de actividades y tareas, el aburrimiento, la desilusión y el miedo.

En su estudio exploratorio con 788 docentes universitarios de México, Sánchez Mendiola et al. (2020) mencionan que el personal docente considera adecuado el apoyo institucional. Sin embargo, existen diferentes tipos de problemáticas como el manejo de tiempo, los horarios de clases, los espacios físicos, la comunicación institucional, el acceso a Internet, la disposición de equipos de cómputo, los conocimientos de plataformas educativas, el manejo de grupos a distancia, la evaluación del estudiantado, la actitud y la disposición del estudiantado, la frustración, el cansancio y la ansiedad.

En su investigación sobre la adaptación de los cursos en una universidad brasileña, Parot de Sousa y Voos Kaspary (2020) encuentran que hubo una adaptación improvisada del personal docente a la virtualidad, ya que este último tuvo que transformar su casa en oficina, 
afrontar las dificultades de la planificación de un curso a distancia en un lapso corto. En su investigación, realizada en Argelia con 2000 docentes universitarios de diversos campos, Lassassi, Lounici, Sami, Tidjani y Benguerna (2020) encuentran que el personal docente ha identificado diversas dificultades como la ausencia de contacto directo con el estudiantado, los problemas de conectividad a Internet, las malas condiciones de vida, los problemas de adaptabilidad de los materiales del curso, la falta de conocimientos tecnológicos y la incertidumbre del futuro.

Louiz (2020), en un estudio realizado en Marruecos, identifica que el cuerpo docente siente que la enseñanza a distancia es difícil, ya que no reemplaza los cursos presenciales, pero es la mejor solución para estas circunstancias. Alladatin, Gnanguenon, Borori y Fonton (2020) coinciden con las personas investigadoras precedentes y agregan también que la falta de formación y de acompañamiento al personal docente es un gran inconveniente para que este proceso sea exitoso. Además, recomiendan que la enseñanza a distancia debe fomentar la autonomía y la motivación del estudiantado, y que tenga acceso a los recursos utilizados en el curso. En su investigación cualitativa sobre la percepción de 10 docentes de Chile, RamosHuenteo, García-Vásquez, Olea-González, Lobos-Peña y Sáez-Delgado (2020) identifican que el profesorado tuvo un papel primordial en las dimensiones emocional y académica del estudiantado, ejerciendo un doble rol: psicólogo y educador. Además, el profesorado experimentó pena, angustia, miedo, estrés e incertidumbre y un aumento considerable del trabajo docente.

Existen también investigaciones sobre la enseñanza a distancia en periodo de pandemia en otros idiomas, como el caso de Macintyre, Gregersen y Mercer (2020), quienes realizaron una investigación cuantitativa con 600 docentes de lengua. Estas personas investigadoras identifican que el estrés del profesorado se debe a la combinación de tareas domésticas y académicas, a la jornada irregular del trabajo, a la falta de actividades recreativas y a la adaptación repentina de la modalidad presencial a la enseñanza remota. En su investigación con el profesorado de inglés, Sánchez García (2020) concluye que la creatividad fue uno de los factores que más ha influenciado al personal docente durante la adaptación a la enseñanza remota. En una investigación cualitativa con 18 docentes de inglés en Indonesia, Lie et al. (2020) encuentran que el mayor desafío del profesorado es aprender a utilizar diversas herramientas tecnológicas para dar continuidad al proceso educativo, dedicar más tiempo a la preparación y a las consultas del estudiantado, la limitación de tiempo de la plataforma Zoom. 
En su investigación cualitativa, por medio de un cuestionario, Lestiyanawati y Widyantoro (2020) mencionan que la dificultad mayor de las clases en línea es el acceso a Internet, sobre todo, cuando se da la clase por Zoom. Rescatan también que el factor económico del estudiantado es un inconveniente para un proceso educativo óptimo. En su investigación cualitativa con tres docentes de inglés, Gao y Zhang (2020) coinciden con las personas autoras precedentes y agregan que la ventaja de la enseñanza en línea es la disponibilidad de los materiales para el estudiantado, ya que puede revisarlos en cualquier momento y las veces que quiera. Implica también un cambio de rol de la persona docente, ya que pasa de ser un transmisor de conocimientos a guía del proceso de aprendizaje, otorgando de esta manera una mayor autonomía al estudiantado.

Con respecto a las investigaciones sobre el caso del francés, El Mousadik y Abentak (2020) explican su experiencia de la enseñanza del francés a un grupo de 14 pasantes alófonos durante la pandemia. Dentro de los principales resultados obtenidos se encontró que el cambio a la enseñanza remota trajo diferentes desventajas como la falta de productividad y de concentración durante el curso por parte del estudiantado, los problemas de conexión, la calidad del sonido, la falta de interacción entre el estudiantado para la producción oral. A pesar de estas dificultades, se logró mantener su atención durante el curso, realizando varias adaptaciones como preguntar sobre su situación durante el confinamiento, ser más flexible con la fecha de entrega de los trabajos o tareas o proponer una atención más individualizada.

Por su parte, Lollia e Issaieva (2020) realizaron una investigación cualitativa con siete docentes en el contexto de la Isla de Guadalupe, coinciden con El Mousadik y Abentak (2020) sobre el acceso a Internet y el acceso a material informático y la falta de contacto directo con el estudiantado. Agregan, también, la sobrecarga de trabajo y la falta de tiempo para la planificación. Además, el profesorado indica que la falta de apoyo y de acompañamiento de la administración para asegurar la continuidad pedagógica. Para la continuidad pedagógica, el cuerpo docente emplea el correo, el teléfono celular, Pronote, Whatsapp, Zoom, entre otros. Encuentran también que las personas docentes no variaron sus prácticas evaluativas, ya que eran las mismas antes y durante la pandemia. Finalmente, Louiz (2020) identifica que el personal docente encuentra que la enseñanza a distancia ha sido difícil, ya que no reemplaza el curso en presencial, además considera que esta modalidad es pasajera y que fue impuesta para continuar con la enseñanza en los cursos.

Por lo tanto, enseñar una lengua extranjera en un contexto exolingüe representa un reto, aún más a nivel universitario en el que se propone una formación de base para futuros 
profesionales de la enseñanza del francés y especialistas de la lengua francesa. Este aspecto, aunado a los retos que conlleva una enseñanza virtual improvisada, inciden en el ejercicio de la profesión de las personas docentes de francés de la UCR, razón por la cual es relevante que se analicen sus percepciones sobre la virtualidad y sobre cómo se vivió esta experiencia. En correspondencia con esta problemática en la que las personas docentes son protagonistas, esta investigación propone analizar cuáles fueron las percepciones de las personas docentes durante el año 2020.

En consecuencia, describir y analizar estas percepciones constituye un ejercicio necesario para hacer un balance sobre cómo el personal docente enfrentó el curso lectivo 2020 y cuáles fueron sus percepciones acerca de esta experiencia detrás de la pantalla. Por otro lado, desde una perspectiva didáctica, una investigación de este tipo, permite reflexionar y debatir acerca de la relevancia y pertinencia tanto del modelo educativo vigente antes de la pandemia en el área de francés como de las prácticas docentes y la modalidad de los cursos.

\section{Referente teórico}

En esta sección, se presentan los diferentes elementos teóricos que se utilizarán en la investigación. Primeramente, se explica el concepto de la enseñanza remota de emergencia. Luego, se expone el concepto de las TIC en la enseñanza remota y, por último, se termina este apartado con la definición de percepciones.

\subsection{La enseñanza remota de emergencia}

La enseñanza en línea ha sido una tendencia en la enseñanza de lenguas desde hace ya varios años. Los modelos de enseñanza a distancia o en línea tienen un denominador común, la planificación y el diseño de dispositivos creados para estas modalidades. En situaciones de emergencia como la desatada por la pandemia del COVID-19, no se puede referir al modelo adoptado como enseñanza a distancia o en línea, ya que, en este contexto en particular, la persona docente, responsable en primera línea del proceso de enseñanza y aprendizaje, no contó con el tiempo ni la preparación para impartir sus cursos.

Esta discusión es puesta sobre la mesa por un grupo de investigadores estadounidenses (Hodges, Moore, Lockee, Trust y Bond, 2020), quienes señalan que en circunstancias tan particulares como las del 2020, se buscó un acceso temporal a la instrucción, es decir, que en cuanto las circunstancias lo permitan, habrá un inminente regreso a las aulas. Contrariamente al curso en línea que pasa por distintas fases de creación, diseño y planificación, en el 2020 
se apostó por una adaptación improvisada a la virtualidad, no se planteó como una opción, sino que fue una imposición a nivel institucional y nacional como resultado de la situación sanitaria mundial.

Tomando en cuenta este contexto, Hodges et al. (2020) acuñaron el término enseñanza remota de emergencia. En cuanto a la elección de los conceptos que la componen, aclaran que "enseñanza" hace referencia no solo a la práctica o profesión de una persona docente, sino que se refiere particularmente a su rol durante el contexto de la pandemia y su impacto en la primera línea de acción como "las tareas realizadas durante los cambios de emergencia cumpliendo un rol de maestro / instructor / profesor" (p. 2). En esta definición destaca el valor de la creatividad, de la adaptabilidad y la constante búsqueda del bienestar de las personas estudiantes. Es importante señalar, además, que este enfoque fue el único modelo adecuado para no detener la labor educativa en muchos países, como lo mencionan Prieto Urgilés, Rodríguez Cajamarca y Verdugo González (2020) en su investigación. Por lo anterior, en esta investigación se adoptará dicho término para referirnos a las circunstancias del 2020, ya que Costa Rica no fue la excepción. Asimismo, se alternará con el término de curso virtual, que se refiere al uso de la tecnología como medio para impartir cursos.

\subsection{Las TIC en la enseñanza remota de emergencia}

En el ámbito educativo, las tecnologías de la información y comunicación (TIC) pueden ser definidas como un recurso al servicio del aprendizaje que está, además, compuesto:

[...] por herramientas ofimáticas (procesadores de textos, hojas de cálculo), editores de páginas, el uso de vídeo, audio, bases datos, medios de comunicación síncronos y asíncronos (los chats, foros, correos electrónicos), materiales multimedia, pizarras electrónicas, carteleras, bibliotecas virtuales, páginas web y otros. (Marqués Graells, 2013, p. 8)

Se debe tomar en cuenta que, aunque los recursos tecnológicos actualmente están a solo un clic, es común que en un mismo grupo de docentes se encuentren en distintos niveles de fluidez digital (Hodges et al, 2020). Por lo tanto, en un contexto de emergencia, el uso de la tecnología en la enseñanza se dio de manera heterogénea.

En el contexto inusual de la pandemia, las personas docentes se convirtieron en pocos días en técnicos expertos en el uso de plataformas y herramientas tecnológicas. Las capacitaciones propuestas a nivel institucional y de la ELM se multiplicaron y fue un periodo 
donde, en muy poco tiempo, nuevos términos se volvieron parte de nuestra vida cotidiana: sincrónico, asincrónico, zoom, entre otros. En el caso de la UCR, las políticas institucionales invitaron al personal docente a usar la plataforma Mediación virtual (MV), así como el correo institucional como principales herramientas para la virtualidad. La ELM sugirió combinar la enseñanza asincrónica con encuentros sincrónicos para poder darle seguimiento al estudiantado.

\subsection{Las percepciones y la docencia}

La percepción es una parte esencial de la conciencia humana y constituye la realidad como ha sido experimentada, es decir, es el resultado del procesamiento de información que consta de estimulaciones a receptores en condiciones que, en cada caso, se deben parcialmente a la propia actividad del sujeto (Arias Castilla, 2008). Dember y Warm (1990), Rosales Sánchez (2015) y Serrahima (2015) consideran que las percepciones son experiencias privadas por las personas. Además, la percepción es vista como un proceso continuo de formación de representaciones mentales (Oviedo, 2004). Vargas Melgarejo (1994) agrega que la percepción está relacionada con la visión del mundo de los grupos sociales y depende de los estímulos físicos y sensaciones involucrados. De esta manera, Vargas Melgarejo la define como un proceso cognitivo de la conciencia que consiste en el reconocimiento, la interpretación y la significación con el fin de elaborar juicios en torno a las sensaciones del ambiente físico y social.

Por lo tanto, se puede afirmar que la percepción es una imagen o una representación mental de un individuo formada a partir de las experiencias y de las necesidades producidas por un proceso de selección, interpretación y corrección de sensaciones experimentadas y vividas. La percepción, por su naturaleza, es algo subjetivo, ya que puede variar de una persona a otra de acuerdo con los estímulos recibidos. Además, es algo que responde a un fenómeno a corto plazo, puesto que su percepción puede cambiar a medida que enriquece sus experiencias y sus motivaciones.

\section{Metodología}

En esta sección, se explica la metodología utilizada en la investigación. Primeramente, se explica el tipo de investigación. Luego, se presentan el contexto, así como las características de las personas participantes. También se va a tratar el instrumento de 
recolección de datos, así como su procedimiento. Finalmente, se describe el tipo de análisis realizado.

\subsection{Enfoque}

Esta investigación tiene la característica de una investigación cualitativa de tipo exploratorio, ya que busca descubrir, explorar y comprender un fenómeno poco conocido o estudiado (Fortin, 2010). Además, se busca comprender el fenómeno desde el punto de vista de las personas participantes en la investigación, lo que permite conocer y comprender los significados que atribuyen a las experiencias vividas (Fortin, 2010; Lessard-Hébert, Goyette y Boutin, 1996).

\subsection{Participantes y contexto de la investigación}

Las personas participantes en esta investigación son 19 docentes de la sección de francés de primer año, de II a IV año de la carrera de Bachillerato en Francés y de Francés para otras carreras de la UCR. Participaron 11 mujeres $(57,9 \%)$ y 8 hombres $(42,1 \%)$. Esto equivale a un 63,3\% del total del personal docente. El 68,4 \% de las personas participantes tiene entre 6 a 15 años de experiencia en el campo de la enseñanza del francés y áreas afines (lingüística, enseñanza de la lengua, literatura, cultura, historia, fonética). En la Figura 1, se presenta la información detallada sobre los años de experiencia de las personas participantes:

\section{Figura 1}

Años de experiencia de las personas participantes, I ciclo 2020

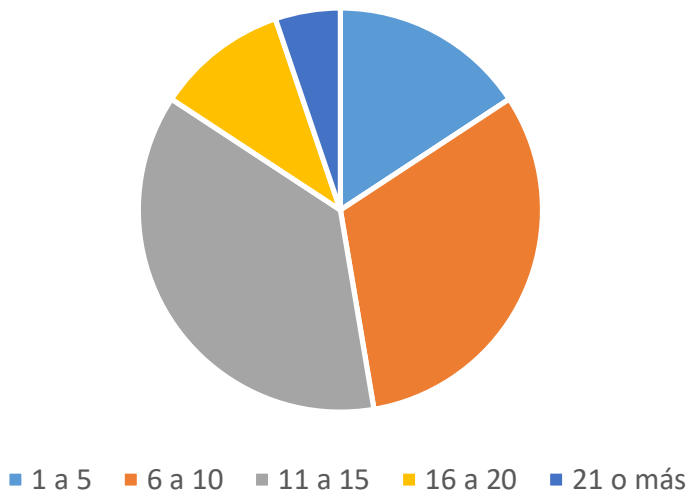

Fuente: Elaboración propia de las personas autoras

La edad promedio del personal docente es de 30 a 50 años. En la Figura 2, se presenta la información detallada sobre el rango de edad. 
Figura 2

Rango de edad de las personas participantes, I ciclo 2020

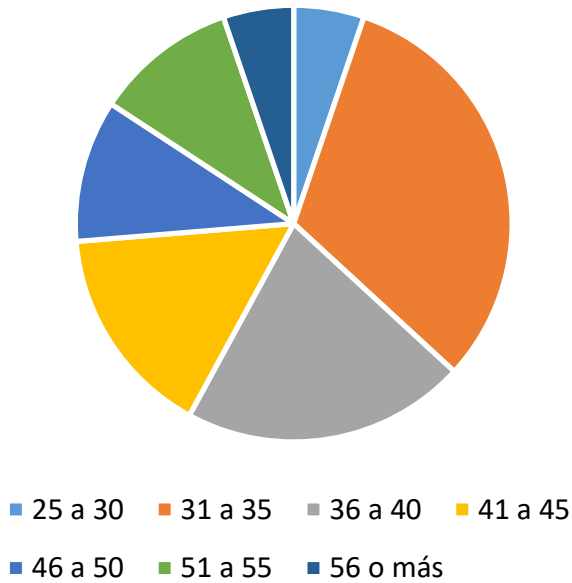

Fuente: Elaboración propia de las personas autoras

Para formar parte de esta investigación, se consideró como criterios de inclusión los siguientes: docentes de francés en la Universidad y haber impartido algún curso durante el contexto de la pandemia del COVID-19 en el primer semestre del 2020. El consentimiento informado se envió por medio de correo electrónico para solicitar la participación del personal docente durante el mes de mayo de 2021.

\subsection{Técnicas de recolección de datos}

El instrumento empleado para la recolección de datos fue un cuestionario aplicado en línea a las personas participantes. El cuestionario está compuesto de las siguientes partes: información personal de las personas participantes (seis preguntas cerradas), experiencia con respecto a impartir cursos virtualmente (seis preguntas cerradas y cuatro abiertas), percepción sobre la vivencia con respecto a la virtualidad de los cursos durante el primer semestre del año 2020 (diez preguntas abiertas) y expectativas sobre capacitaciones para prepararse a la virtualidad de los cursos (tres preguntas abiertas).

El instrumento fue aplicado durante el mes de julio de 2020. Antes de la aplicación del instrumento, se realizó su validación por tres personas expertas y también se aplicó a una persona participante para ver si este permitía recoger los datos necesarios a la investigación. Esta etapa se realizó a finales de mayo y principios de junio de 2020. Se hicieron ajustes y modificaciones al instrumento antes de aplicarlo a la población participante. El instrumento está compuesto por preguntas abiertas en su mayoría y algunas preguntas cerradas. Se utilizó la aplicación de Google forms para crear el instrumento y luego, se envió el enlace por correo 
a las personas docentes que manifestaron interés en participar en la investigación. Se dejó abierto el instrumento durante todo el mes de julio y principios de agosto de 2020 para que el personal docente respondiera.

\subsection{Análisis de los resultados}

Para proceder a la etapa de análisis de los resultados, se realizó un análisis de los contenidos de acuerdo con la matriz de categoría establecida previamente en la investigación. Para la matriz de categoría, se emplearon los siguientes códigos para clasificar la información obtenida: experiencia, recursos, herramientas, capacitación. A su vez, estas grandes categorías se dividen en subcategorías como, por ejemplo, la categoría 'experiencia' tiene los siguientes códigos: ventajas (académica y desplazamiento) y dificultades (logística, socioafectiva, pedagógica y tecnológica). Se utilizó la herramienta QDA Miner para el análisis de la información. La información obtenida en las categorías de recursos, herramientas y capacitación será objeto de análisis para otro artículo.

\section{Descripción y análisis de los resultados}

En esta sección se presentan los resultados de acuerdo con las categorías predeterminadas previamente.

\subsection{Percepciones y uso general de las TIC}

En los datos arrojados por la primera parte del cuestionario se abordan temas relacionados con el uso de TIC y acerca de las percepciones sobre este tema. Casi la mitad de las personas participantes $(47,4 \%)$ ha tenido experiencia impartiendo cursos a distancia (en la UCR y en otras universidades estatales) y solamente una persona docente ha impartido cursos virtuales en otra institución. En la Figura 3, se ilustra la distribución del personal docente según su experiencia impartiendo cursos en modalidad a distancia. 
Figura 3

La distribución del personal docente impartiendo cursos en la enseñanza remota, I ciclo 2020

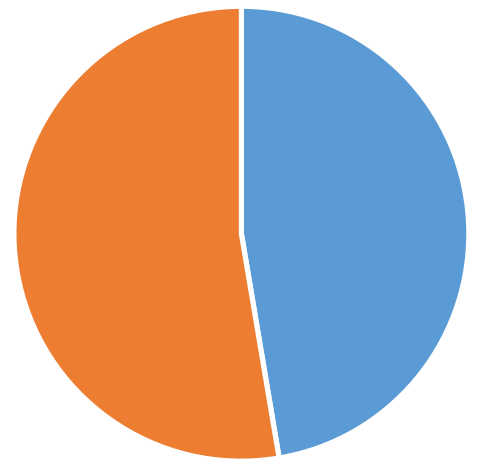

- Con experiencia impartiendo cursos bimodales

- Sin experiencia impartiendo cursos bimodales

Fuente: Elaboración propia de las personas autoras.

En cuanto a la plataforma institucional MV, la mayoría de las percepciones $(57,8 \%)$ son positivas. Muchos consideran que es un recurso excelente, práctico y flexible como lo ilustra el siguiente verbatim: "Ha sido muy práctico y permite organizar todo el material en un mismo lugar...con los cambios que le hicieron se mejoró bastante la plataforma" (P1, comunicación personal, 01 de julio del 2020). Algunas personas docentes (31,5\%) consideran que aún debe haber mejoras en cuanto a la velocidad y que se deben proponer más capacitaciones para su uso óptimo: "bien, pero hay que formarse aún más en su uso" ( $\mathrm{P} 12$, comunicación personal, 07 de julio del 2020). Adicionalmente, algunas percepciones negativas (10,5\%) que se atribuyen a esta herramienta son que presenta poca variedad de ejercicios para los exámenes, que es rígida, lenta y que no carga los documentos rápidamente, por ejemplo: "es un poco lenta y subir archivos es un poco complicado si se compara con otras plataformas más ágiles y flexibles" (P19, comunicación personal, 08 de agosto del 2020).

Cuando se les consultó acerca del uso que se le dio a la plataforma durante el I ciclo 2020 , el $78,9 \%$ de personas participantes indican que usaron la plataforma para envío de tareas o asignaciones o para depositar material para el curso. Un 47,3\% indicó que proponía ejercicios en línea y un 31,5\% señaló el uso del foro. En la Tabla 1, se detallan los usos que se le ha dado a la plataforma por el personal docente. 
Tabla 1

Uso dado a la plataforma de Mediación Virtual, I ciclo 2020

Tipo de uso Cantidad y porcentaje de docentes

Foro

Envío de tareas o trabajos

Depósito de materiales para el curso

Ejercicios o exámenes en línea

Wiki
$31,5 \%(\mathrm{~N}=6)$

$78,9 \%(\mathrm{~N}=15)$

$78,9 \%(\mathrm{~N}=15)$

$47,3 \%(\mathrm{~N}=9)$

$10,5 \%(\mathrm{~N}=2)$

Fuente: Elaboración propia de las personas autoras

En relación con las herramientas tecnológicas usadas por las personas docentes, las más mencionadas fueron Youtube, Zoom y Whatsapp. En cuanto a los recursos tecnológicos que más usaron para crear contenido, mencionaron la creación de audios y de vídeos o uso de Powerpoint o uso de videos de Youtube. En la Tabla 2, se presentan los recursos tecnológicos que utilizaron para crear contenido de sus cursos:

Tabla 2

Recursos usados para crear contenido de sus cursos, I ciclo 2020

\begin{tabular}{lc}
\hline \multicolumn{1}{c}{ Tipo de recursos empleados } & Cantidad y porcentaje de docentes \\
\hline Creación de audios & $52,6 \%(\mathrm{~N}=10)$ \\
Creación de videos & $47,3 \%(\mathrm{~N}=9)$ \\
Quizlet & $26,3 \%(\mathrm{~N}=5)$ \\
Kahoot & $26,3 \%(\mathrm{~N}=5)$ \\
Vocaroo & $26,3 \%(\mathrm{~N}=5)$ \\
Screencast-O-Matic & $31,5 \%(\mathrm{~N}=6)$ \\
Padlet & $31,5 \%(\mathrm{~N}=6)$ \\
Drive & $42,1 \%(\mathrm{~N}=8)$ \\
Youtube & $57,8 \%(\mathrm{~N}=11)$ \\
Google Classroom & $31,5 \%(\mathrm{~N}=6)$ \\
Powtoon & $10,5 \%(\mathrm{~N}=2)$ \\
WhatsApp & $31,5 \%(\mathrm{~N}=6)$ \\
Powerpoint & $52,6 \%(\mathrm{~N}=10)$ \\
Canvas & $0(\mathrm{~N}=0)$ \\
\hline
\end{tabular}

Fuente: Elaboración propia de las personas autoras

\subsection{Percepciones sobre las ventajas que ofreció la enseñanza remota de emergencia}

En este apartado, se muestran las diferentes ventajas encontradas en la investigación, las cuales se dividen en dos grandes grupos: las ventajas académicas y las ventajas de quedarse en casa. 


\subsubsection{Ventajas académicas}

A pesar de que la situación educativa del 2020 fue una consecuencia directa de la pandemia y de que, en el área de francés no se ofrecían cursos virtuales, todo proceso de enseñanza es susceptible de suscitar reflexión, aún más al tratarse de la modalidad remota de emergencia. En lo concerniente a las ventajas que presentó esta modalidad, ninguna persona participante mencionó ventajas en el ámbito académico para la población docente, todas recaen en el estudiantado como se ilustra en la Tabla 3.

Tabla 3

Ventajas académicas de la virtualidad para el estudiantado, I ciclo 2020

Tipo de ventaja

Accesibilidad y disponibilidad del material

Autonomía del estudiantado

Seguimiento individual del estudiantado

Adaptación al ritmo de aprendizaje de cada estudiante

Fuente: Elaboración propia de las personas autoras
Cantidad y porcentaje de docentes

$42,1 \%(\mathrm{~N}=8)$

$21,05 \%(\mathrm{~N}=4)$

$21,05 \%(N=4)$

$21,05 \%(\mathrm{~N}=4)$

Como se ilustra en la Tabla 3, las personas docentes insisten en que uno de los aspectos más positivos para el estudiantado fue la disponibilidad inmediata del material de los cursos, por ejemplo: "El estudiante tiene acceso a todo el material del curso, lo puede consultar cuando quiera" (P2, comunicación personal, 01 de julio del 2020). Señalan también que el hecho de proponer trabajo asincrónico permitió que el estudiantado pudiera cumplir con los objetivos respetando su ritmo de aprendizaje: "El hecho de proponer cursos asincrónicos también es positivo, cada quien puede ir a su ritmo" (P2, comunicación personal, 01 de julio del 2020).

Además, otro aspecto mencionado fue que la virtualidad presenta grandes ventajas para centrar el aprendizaje en el estudiantado. Se menciona, por ejemplo, que la virtualidad motiva a que el estudiantado sea autónomo: "la ventaja es que hay más autonomía del estudiante" (P1, comunicación personal, 01 de julio del 2020). Según algunas personas docentes $(21,05 \%)$, la corrección individual fue más detallada y el seguimiento de las personas estudiantes fue más significativo que en semestres anteriores en la modalidad presencial: "He podido hacer mucha corrección específica, mucho ejercicio de autonomía, de lectura, entre otros" (P12, comunicación personal, 07 de julio del 2020). 


\subsubsection{Ventajas derivadas de quedarse en casa}

Otra percepción mencionada por el personal docente $(42,1 \%)$ fue que, gracias a la virtualidad, las personas estudiantes y docentes no deben desplazarse al campus, lo que incide en un mejor aprovechamiento del tiempo: "Hay mejor asistencia y puntualidad, pues los estudiantes no deben desplazarse" (P10, comunicación personal, 06 de julio del 2020). Una persona docente mencionó que no desplazarse al campus ha hecho que no se pierda tiempo a causa del tráfico y además, es una solución para el problema que representaba encontrar lugar en los estacionamientos del campus: "No hay desplazamiento físico, no hay presas, el estrés se traduce en otro tipo" (P14, comunicación personal, 07 de julio del 2020).

\subsection{Percepciones sobre las dificultades de la enseñanza remota de emergencia}

En este apartado se presentan las dificultades que ha tenido el personal docente en cuanto a la virtualidad, estas se presentan en cuatro grandes categorías de dificultades según lo propuesto por Sánchez Mendiola et al. (2020): dificultades logísticas, dificultades tecnológicas, dificultades pedagógicas y dificultades socioafectivas.

\subsubsection{Dificultades logísticas}

Las dificultades logísticas se refieren a aquellas relacionadas con el equipo de trabajo (computadora, muebles y espacio físico) necesario para el teletrabajo, al manejo del tiempo y a todo lo relativo a la comunicación institucional. En la Tabla 4 se resume la distribución de las dificultades logísticas.

Tabla 4

Las dificultades logísticas de la virtualidad, I ciclo 2020

\begin{tabular}{lc}
\hline \multicolumn{1}{c}{ Tipo de dificultad logística } & Cantidad y porcentaje de docentes \\
\hline El manejo de tiempo & $36,8 \%(\mathrm{~N}=7)$ \\
La comunicación institucional & $21,05 \%(\mathrm{~N}=4)$ \\
El equipo de trabajo del profesorado & $10,5 \%(\mathrm{~N}=2)$ \\
El equipo de trabajo del estudiantado & $26,3 \%(\mathrm{~N}=5)$ \\
\hline
\end{tabular}

Fuente: Elaboración propia de las personas autoras.

El tiempo es la dificultad que más se mencionó por parte de las personas docentes. Señalan que el tiempo de preparación de los cursos y consignado a la corrección de asignaciones se duplicó y en algunos casos se triplicó, lo que ha causado fatiga extrema: "La multiplicación de trabajo de preparación y corrección. Además de subir todo en PDF corregido a la plataforma" (P9, comunicación personal, 06 de julio del 2020) y "El manejo del tiempo, pues la preparación toma muchísimo más tiempo, así como la corrección de trabajos y preparación de exámenes" (P11, comunicación personal, 07 de julio del 2020). 
Otra dificultad mencionada por el personal docente fue la falta de equipo adecuado del estudiantado para las clases sincrónicas: “...También el problema reside en que no todos los estudiantes tienen acceso a Internet de buena calidad o a dispositivos que les permitan participar en las sesiones sincrónicas" (P2, comunicación personal, 01 de julio del 2020). En el caso del personal académico, hubo también algunas personas docentes que mencionaron que no contaba con un equipo adecuado en su hogar para las clases: “... Además el no contar con todo el equipo digital dificulta el trabajo" (P18, comunicación personal, 15 de julio del 2020).

Dentro de estas dificultades figuran también las percepciones relativas a la manera en cómo la UCR respondió ante esta situación de emergencia. Según lo expresan las personas docentes, una de las mayores dificultades es la falta de alfabetización tecnológica de las personas estudiantes, por lo que sugieren que la institución haga esfuerzos en este sentido, en caso de que la enseñanza remota se mantenga: "Falta de conectividad e insuficiente alfabetización en virtualidad" (P8, comunicación personal, 06 de julio del 2020). Asimismo, creen necesaria una directriz institucional en la cual se establezca que encender la cámara sea obligatorio durante las sesiones sincrónicas: “...tengo alumnos que nunca encendieron ni cámara ni micrófono por lo que no sé si estuvieron atentos o no a las clases. Debería haber un comunicado de la Escuela solicitando la obligatoriedad de poner la cámara" (P16, comunicación personal, 13 de julio del 2020). También, varias personas docentes comentan que al inicio del semestre hubo problemas con el correo institucional y con MV y que dichosamente pudieron ser superadas con el avance del semestre: "al inicio hubo problemas con el correo y la plataforma" (P5, comunicación personal, 06 de julio del 2020).

\subsubsection{Dificultades tecnológicas}

Las últimas dificultades logísticas mencionadas en el párrafo anterior pueden relacionarse también con dificultades tecnológicas, sin embargo, debido a que la falla detectada por las personas docentes está mediada por la Institución, se indicó en el apartado anterior. En la Tabla 5 se ilustran algunas dificultades tecnológicas mencionadas por el personal docente.

Tabla 5

Dificultades tecnológicas de la virtualidad, I ciclo 2020

\begin{tabular}{lc}
\hline \multicolumn{1}{c}{ Tipo de dificultad tecnológica } & Cantidad y porcentaje de docentes \\
\hline La conexión a Internet del estudiantado & $21,05 \%(\mathrm{~N}=4)$ \\
La conexión a Internet del profesorado & $10,5 \%(\mathrm{~N}=2)$ \\
El manejo de plataformas institucionales & $5,2 \%(\mathrm{~N}=1)$ \\
El manejo de herramientas tecnológicas & $21,05 \%(\mathrm{~N}=4)$ \\
\hline
\end{tabular}

Fuente: Elaboración propia de las personas autoras 
Las dificultades tecnológicas tienen que ver, sobre todo, con la conexión a Internet en general, con el acceso a equipos de cómputo, a dispositivos electrónicos, con el conocimiento de la plataforma y con problemáticas más específicas relacionadas a lo mencionado: "Si el estudiante no cuenta con computadora y buena conexión a Internet es difícil que se pueda unir a las clases" (P19, comunicación personal, 08 de agosto del 2020).

Cuatro docentes señalaron que la ELM y la UCR propusieron capacitaciones y pusieron a disposición del profesorado tutoriales para el uso de herramientas tecnológicas, pero sin que se diera un acompañamiento real. Dos participantes insisten en que tuvieron problemas de conexión durante el semestre y de Internet en general, lo que provocó comentarios desagradables por parte de colegas y, por ende, aún más estrés y cansancio. Asimismo, se reportan gastos imprevistos debido a la compra de equipo de cómputo y mobiliario adecuado para el teletrabajo.

\subsubsection{Dificultades pedagógicas}

Muchas de las dificultades mencionadas tienen que ver con el estudiantado y con su aprendizaje durante la pandemia. Una buena parte de las personas docentes $(47,3 \%)$ insiste en que una de las mayores dificultades que encontraron fue la influencia del contexto del estudiantado en el aprendizaje en la modalidad remota: "El presupuesto de muchos o las particularidades de la vida personal (muchos conectados en la casa, ruido y vida de familia se mezclan" (P14, comunicación personal, 07 de julio del 2020). Esto se traduce como la influencia negativa que ejercieron los problemas familiares y económicos en la experiencia de aprendizaje. Asimismo, se citan varias veces problemas de concentración del estudiantado durante las sesiones sincrónicas, debido, por ejemplo, al ruido o al hecho de convivir con familiares que interrumpían sus clases.

Otro aspecto que, según las personas participantes, incidió negativamente en el proceso de aprendizaje del estudiantado fue el hecho de no contar con dispositivos electrónicos adecuados para los cursos virtuales, así como el mal funcionamiento de micrófonos y cámaras. Algunas personas docentes mencionan que hubo estudiantes que trabajaron con su celular o con dispositivos prestados. Otra de las mayores problemáticas mencionadas durante el primer semestre de enseñanza remota de emergencia fue indudablemente la mala conexión a Internet de algunas personas estudiantes.

El $31,5 \%$ de las personas docentes señalan que el hecho de que no se enciendan las cámaras en las sesiones sincrónicas es, por una parte, desgastante, pues no permiten 
establecer una comunicación e interacción efectivas durante las clases: "Poca interacción de los estudiantes entre ellos y con el docente" (P17, comunicación personal, 14 de julio del 2020). Además, opinan que con las cámaras apagadas es difícil confirmar el nivel de implicación del estudiantado y que no hay manera de comprobar si en realidad las personas estudiantes están siguiendo la clase o están llevando a cabo otras actividades en vez de participar y estar atentos durante la sesión sincrónica: “...no ponen el video o foto por lo que dificulta saber si están en la clase o poniendo atención" (P18, comunicación personal, 15 de julio del 2020).

En relación con la experiencia de enseñanza en la modalidad remota de emergencia , la mayoría del personal docente $(57,8 \%)$ indica que detectaron también falta de interés, compromiso y poca participación por parte del estudiantado: "Falta de interés del estudiantado" (P4, comunicación personal, 07 de junio del 2020), "la falta de interacción directa y espontánea afecta los procesos" (P10, comunicación personal, 06 de julio del 2020), "es difícil, sobre todo por el tema de compromiso por parte de los estudiantes, a veces no se conectan, a veces no quieren utilizar la cámara" (P19, comunicación personal, 08 de agosto del 2020). Así mismo, mencionan que hubo poca interacción entre compañeros de clase, aspectos que también pudieron influenciar el aprendizaje durante este ciclo.

\subsubsection{Dificultades socioafectivas}

Este tipo de dificultad tiene que ver con las percepciones sobre aspectos emocionales y físicos que vivieron las personas docentes. Cabe mencionar que estas fueron las más recurrentes en las respuestas abiertas del cuestionario. En la Tabla 6 se resumen las principales dificultades socioafectivas mencionadas por el personal docente.

Tabla 6

Dificultades socioafectivas, I ciclo 2020

\begin{tabular}{lc}
\hline \multicolumn{1}{c}{ Tipo de dificultad socioafectiva } & Cantidad y porcentaje de docentes \\
\hline El estrés & $26,3 \%(\mathrm{~N}=5)$ \\
El cansancio o la fatiga & $26,3 \%(\mathrm{~N}=5)$ \\
El desgaste físico y mental & $21,05 \%(\mathrm{~N}=4)$ \\
La frustración & $21,05 \%(\mathrm{~N}=4)$ \\
La tristeza & $5,2 \%(\mathrm{~N}=1)$ \\
El miedo & $10,5 \%(\mathrm{~N}=2)$ \\
La ansiedad & $5,2 \%(\mathrm{~N}=1)$ \\
El insomnio & $5,2 \%(\mathrm{~N}=1)$ \\
La falta de contacto humano & $26,3 \%(\mathrm{~N}=5)$ \\
La inseguridad & $26,3 \%(\mathrm{~N}=5)$ \\
\hline
\end{tabular}

Fuente: Elaboración propia de las personas autoras 
Las personas docentes consideran que la enseñanza remota de emergencia causó estrés, desgaste, cansancio extremo, frustración, tristeza, incertidumbre, miedo, insomnio y ansiedad. En consecuencia, se desencadenaron problemas físicos como el dolor de espalda y problemas de la vista (ceguera nocturna, agravamiento de condiciones existentes antes de la pandemia): "Mayor fatiga al dar las clases en una única posición" (P10, comunicación personal, 06 de julio del 2020). Dos personas docentes indicaron que su vida familiar y personal se vio afectada debido al teletrabajo; otras mencionan que un elemento que empeoró su estrés fue que las personas estudiantes no encendían la cámara durante las sesiones sincrónicas y a veces tampoco encendían los micrófonos. Asimismo, otra desventaja que incide directamente en la dimensión socioafectiva fue la inversión económica que se tuvo que asumir como producto de la pandemia. Dentro de los principales gastos el figuran la compra de equipo de cómputo (UPS, impresora, computadora), así como cambio de banda de Internet para tener un mejor servicio: "debe ser que la Universidad vela para que el docente tenga todas las herramientas tecnológicas, ya que es simplemente incomprensible que sea el docente quien tenga que utilizar sus herramientas y pagar Internet entre cosas de su propio bolsillo" (P15, comunicación personal, 15 de julio, del 2020).

\subsection{Percepciones acerca de los cursos que podrían virtualizarse en el futuro}

Pese a que, como se ha mencionado en secciones anteriores, la metodología y modalidad de los cursos del I semestre del 2020 en la UCR obedece a una situación impuesta, se consideró preguntar en el cuestionario la percepción de las personas docentes en cuanto a qué cursos son susceptibles de ser propuestos en modalidad virtual en los años por venir. Cabe destacar que en la ELM desde antes de la pandemia algunos cursos del Bachillerato en inglés se habían propuesto en modalidad virtual, como la traducción y la comprensión de lecturas debido a la naturaleza de estos cursos y a la diversificación del perfil de la población estudiantil (personas que trabajan, que tienen familias que mantener, etc.). En consecuencia, se considera válido interrogar a la población de docentes de francés sobre este aspecto, sobre todo partiendo del hecho de que por primera vez todos los cursos de francés se impartieron en esta modalidad.

En el siguiente cuadro se muestran los cursos mencionados por las personas docentes. No se incluyen todos los cursos que se impartieron ni se especifican las siglas ni nombres, tal y como aparecen en el plan de estudios de la carrera, se tomaron en cuenta únicamente los 
citados en las respuestas abiertas. En la Tabla 7 se presenta el listado de cursos según su adaptabilidad para la enseñanza remota.

Tabla 7

Número de cursos según adaptabilidad para la enseñanza remota, Escuela de Lenguas Modernas, UCR, I ciclo 2020

\begin{tabular}{lc}
\hline \multicolumn{1}{c}{ Cursos más aptos para la virtualidad } & Cantidad de docentes que lo mencionó \\
\hline Francés para otras carreras & $\mathrm{N}=6$ \\
Gramática & $\mathrm{N}=4$ \\
Expresión oral & $\mathrm{N}=2$ \\
Historia o cultura & $\mathrm{N}=2$ \\
Literatura & $\mathrm{N}=1$ \\
Lexicología & $\mathrm{N}=1$ \\
Cursos de licenciatura & $\mathrm{N}=6$ \\
Cursos más aptos para la virtualidad & $\mathrm{N}=5$ \\
Francés Integrado & $\mathrm{N}=2$ \\
Literatura & $\mathrm{N}=1$ \\
Fonética & $\mathrm{N}=1$ \\
Francés para otras carreras & $\mathrm{N}=1$ \\
Gramática & \\
Comprensión de textos en francés &
\end{tabular}

Fuente: Elaboración propia de las personas autoras

Según las percepciones de seis personas docentes, los cursos pertenecientes a la sección de Francés para otras carreras se pueden adaptar fácilmente a la enseñanza virtual. Algunas de las razones que justifican esta elección son la madurez del estudiantado que sigue estos cursos, su responsabilidad y su compromiso.

En la Tabla 7 se puede apreciar que los cursos de gramática también son aptos para ser virtualizados. En este caso en particular, las personas docentes no explican el por qué. En el caso de los demás cursos que se consideran adaptables a la enseñanza remota, las razones que exponen las personas docentes se centran en la percepción de que en cursos como los de Cultura e Historia, se debe leer más material y trabajar de manera autónoma, por lo cual consideran que es más fácil que los cursos se puedan adaptar a la virtualidad.

Además de los datos expuestos en la Tabla 7, tres docentes consideran que todos los cursos pueden ser adaptados a la enseñanza remota. Mencionan que esto sería posible bajo la condición de que se propongan sesiones sincrónicas y asincrónicas; otros indican que sería posible siempre y cuando se cuente con el debido tiempo para planificar y crear estos cursos.

En el caso de los cursos que no son aptos para la enseñanza remota, figura, en primer lugar, el curso Francés Integrado I. Seis docentes consideran que este curso no se debe proponer en modalidad virtual. Una persona comenta que la interacción entre estudiantes y la persona docente es esencial y que en la virtualidad no ha sido efectiva. Otros comentan que 
dado el ritmo y la complejidad del curso (6 créditos, 13 horas semanales) es difícil que se adapte a un curso $100 \%$ virtual. Además de este curso, existen cinco docentes que mencionaron que los cursos de literatura no son adecuados en la virtualidad: "pues no es fácil realizar una práctica conjunta de análisis de textos" (P10, comunicación personal, 06 de julio del 2020).

En cuanto a los demás cursos que no se adaptan a la virtualidad, varias personas docentes tienen la percepción de que cuando se trata de cursos para principiantes, la interacción en modalidad presencial es más necesaria que en cursos de tercer o cuarto año. Asimismo, en el caso de los cursos de fonética, las dos personas que mencionaron que se trata de cursos difíciles de adaptar a la virtualidad, no explican el por qué.

Dos personas docentes indicaron que ninguno de los cursos debería impartirse de manera remota. Una persona señala que "es contradictorio desarrollar el aprendizaje de una lengua extranjera de forma virtual o a distancia" (P15, comunicación personal, 15 de julio del 2020).

Como síntesis, se observa que la enseñanza remota ha traído ciertas ventajas al personal docente como la disponibilidad de los materiales del curso, el evitar desplazarse a la universidad, entre otros. Sin embargo, aparecen también una serie de dificultades como el exceso de trabajo, la conexión a Internet, las interacciones efectivas durante la clase, etc. Además, el personal docente destaca que, a pesar de esta adaptación repentina a la virtualidad, existen algunos cursos que se podrían adaptar a esta modalidad y otros que definitivamente no podrían adaptarse.

\section{Discusión de los resultados}

En los comentarios de las personas docentes es evidente que la pandemia tuvo un gran impacto sobre todo a nivel afectivo, dejando en segundo lugar lo relacionado a lo pedagógico. Cuando se les preguntó cómo se sintieron durante el I ciclo 2020, los términos más recurrentes fueron: cansancio, frustración, agotador, inútil, ansiosa, nerviosa, triste, estrés laboral, miedo, inseguridad. Este fenómeno coincide también con lo identificado en las investigaciones de Silas Casillas y Vázquez Rodríguez (2020), Sánchez Mendiola et al. (2020) y Ramos-Huenteo et al. (2020). Esto se vio reforzado por comentarios como los siguientes: "no me sentí a gusto" (P5, comunicación personal, 06 de julio del 2020), "bien pero no es lo ideal" (P6, comunicación personal, 06 de julio del 2020). Hubo docentes que declararon que el trabajo remoto empeoró ciertas condiciones que ya sufrían antes de la pandemia. Tal es el caso de una docente que 
confirma que "trabajar y crear herramientas y ser bombardeada de mensajes para seguir capacitaciones multiplicaron la ansiedad" (P14, comunicación personal, 07 de julio del 2020). Macintyre et al. (2020) y Sánchez Mendiola et al. (2020) también identificaron esta problemática ya que la jornada irregular y la falta de actividades recreativas son causas que producen o refuerzan la ansiedad. Este tipo de percepciones se pueden explicar mediante el carácter impuesto de la enseñanza remota en el I ciclo del 2020.

Además de las dificultades propias de impartir clases de lengua extranjera en línea, muchos docentes tuvieron que lidiar con problemáticas familiares "en el seno de mi familia no podía interrumpir el ritmo de vida lo que hizo que el ruido de platos, ollas, cocina y televisión a bajo volumen no me dieran claridad de sonido de completa concentración, lo que significaron regaños fuertes para mis hijos para que me dejaran impartir lecciones" (P14, comunicación personal, 07 de julio del 2020). La problemática familiar no es algo propio de los resultados de esta investigación, puesto que otros autores como Silas Casillas y Vázquez Rodríguez (2020) las citan como una dificultad en sus investigaciones.

En algunos casos, como consecuencia de esta situación, las personas docentes sufrieron un deterioro de su salud física tal y como se mencionó en el apartado anterior. Además, varias personas incurrieron en gastos imprevistos para la compra de equipo de cómputo y realizar mejoras en la conexión a Internet, o en modificar espacios en sus casos para darle cabida al teletrabajo, lo que incidió en el ámbito socioafectivo. En el estudio sobre el contexto argelino, Lassassi et al. (2020) mencionan también la problemática de no contar con una conexión estable de Internet, tanto en el caso de docentes como de estudiantes. Una de las personas encuestadas considera lo siguiente en cuanto a los gastos provocados por los cursos impartidos de manera remota: "es simplemente incomprensible que sea el propio docente que tenga que utilizar sus herramientas y pagar Internet entre otras cosas de su propio bolsillo" (P15, comunicación personal, 15 de julio del 2020).

\subsection{Percepciones sobre las ventajas y desventajas de la enseñanza remota de emergencia}

Un gran número de personas docentes considera que la modalidad remota durante el ler semestre 2020 fue un reto y que, pese a su carácter de imposición, sus prácticas metodológicas evolucionaron conforme pasaba el tiempo. Dos personas docentes opinaron lo siguiente: "Bien pero con muchas dudas al principio de cómo impartirlos, ahora con mayor seguridad por la respuesta favorable de los estudiantes" (P18, comunicación personal, 15 de 
julio del 2020), "Al inicio muy nerviosa y con muchísima ansiedad. Con el pasar de los días y ver que al menos en dos de mis tres cursos se iban dando resultados positivos [...] me fui sintiendo mejor" (P11, comunicación personal, 07 de julio del 2021).

Está claro que el ambiente académico durante este periodo crítico estuvo teñido de frustración e incertidumbre. También se presentaron grandes retos en cuanto al uso improvisado y acelerado de nuevas herramientas tecnológicas para adaptarse a la modalidad remota y para lograr que el proceso de enseñanza-aprendizaje fuera lo más eficaz y significativo posible. Esta información coincide con la arrojada en investigaciones como la de Alladatin et al. (2020), Lie et al. (2020) y en la de Parot de Sousa y Voos Kaspary (2020) en donde se señala que la falta de preparación o el poco tiempo que tuvo el personal docente para adaptarse a la enseñanza remota fue un factor determinante en este proceso. Algunas personas docentes informaron que, si bien es cierto que la ELM y la UCR ofrecieron capacitaciones, muchos se sintieron abrumados e incluso bombardeados de información.

Algunos rescatan el hecho de que, a pesar de que la modalidad virtual fue un gran reto, se logró desarrollar el semestre y cumplir con los objetivos planteados antes de la llegada de la pandemia. Recordemos que este ciclo se inició en la modalidad presencial, por lo que algunas personas docentes tuvieron un primer contacto en modalidad presencial con el estudiantado y otros no.

En las respuestas de las personas docentes no se localizó información relativa a las problemáticas relacionadas a la continuidad pedagógica o al seguimiento de las personas estudiantes. Solamente una persona menciona que la virtualidad permite acercarse más al estudiantado por medio de la realimentación de las evaluaciones. A este respecto, Lollia e Issaieva (2020), señalan que, en el contexto de Guadalupe, el seguimiento trató de ponerse en marcha, pero no se desarrolló en las mejores condiciones. Indican que una de las principales fallas fue la formación del cuerpo docente en cuanto a los medios puestos en marcha para asegurar el ritmo de los aprendizajes. Así, las autoras apuntan a que los discursos institucionales y el sentimiento de las personas docentes no siempre coinciden; concluyen que es importante que el personal docente esté preparado para afrontar rupturas y situaciones imprevistas en distintos contextos.

Las autoras afirman también que las personas docentes no creen en el milagro de las soluciones tecnológicas y resaltan la idea de que enseñar no es un milagro, sino más bien un proceso de construcción común. Esta observación sobre el contexto educativo en Guadalupe es similar al de las personas docentes de francés de la UCR. Hay docentes que consideran 
que el uso de la tecnología no es suficiente para asegurar un aprendizaje óptimo. Una persona docente se refiere al uso de la tecnología como un experimento puesto en marcha de un día para otro, otra indica que durante el I ciclo prevaleció un constante "probemos a ver qué" (P7, comunicación personal, 07 de julio del 2020); que se hizo lo que estuvo al alcance y que, a pesar de todo, esta experiencia puede ser valiosa para el futuro.

La capacidad de adaptación en el I ciclo 2020 de algunos se pone de manifiesto en respuestas como las siguientes: "Al principio con mucho miedo, teniendo que adaptar las clases y el material al modo virtual [...]. Sin embargo, durante el transcurso del semestre me fui adaptando" (P3, comunicación personal, 07 de junio del 2020). En cuanto a la flexibilidad y adaptación, se debe mencionar que, si bien es cierto que son dos características esenciales de una persona docente que imparta cursos virtuales, no se puede perder de vista el carácter inusual de la situación que se vivió en el I ciclo 2020. Es importante mencionar que esta flexibilidad del profesorado para con el estudiantado es esencial para llevar a cabo exitosamente esta tarea, como lo afirman Gao y Zhang (2020).

En relación con los inconvenientes, al igual que en los casos expuestos por El Mousadik y Abentak (2020) y Lassassi et al. (2020), en los cursos de francés de la UCR la mayor dificultad percibida por las personas docentes fue la distancia física. Según estas personas autoras, la distancia física impide que se den interacciones y regulaciones pedagógicas. En este sentido, las personas docentes se expresaron acerca de la importancia de la interacción, esencial en el desarrollo de competencias comunicativas y generales del estudiantado. Varios $(26,3 \%)$ señalaron que, aunque haya interacción en las sesiones sincrónicas, la comunicación no es tan eficaz como en la presencialidad. Aunado a esto, citan el problema de las cámaras apagadas y lo frustrante que es dirigirse a una pantalla y a pantallas negras.

Otro inconveniente encontrado fue todo lo relativo al desgaste mental y a las consecuencias físicas del trabajo remoto. Hay docentes que indican que su carga de trabajo se triplicó y otros que pasaron incluso doce horas diarias frente a la pantalla. Este aspecto también fue señalado por otras investigaciones como Lie et al. (2020), Macintyre et al. (2020) y Sánchez Mendiola et al. (2020).

El tema de la evaluación fue mencionado únicamente en tres comentarios. Algunos consideran que la plataforma institucional no ofrece una gama variada de tipos de ejercicio para la creación de exámenes. Dos docentes reconocen que, gracias a esta modalidad remota, hubo mayor seguimiento y retroalimentación con el estudiantado. Esta temática, ampliamente 
tratada por Lollia e Issaieva (2020) fue apenas mencionada por las personas participantes en el presente estudio.

Las dificultades encontradas coinciden con los hallazgos de Sánchez Mendiola et al. (2020) en cuanto a su clasificación. Éstas se clasificaron en logísticas, socioafectivas, tecnológicas y didácticas. Sin embargo, en este estudio las dificultades más recurrentes fueron las logísticas, mientras que en el profesorado de francés de la UCR las más frecuentes fueron las relacionadas a lo socioafectivo, dentro de las que destacan el cansancio extremo y el estrés, relacionados directamente problemas físicos como consecuencia del teletrabajo.

Las ventajas de la virtualidad en el ámbito académico recaen únicamente en el estudiantado. Existe un consenso en cuanto a que lo más positivo de la modalidad virtual es que el estudiantado cuenta siempre con acceso al material del curso, lo que le permite trabajar de manera autónoma. Las personas docentes perciben que el hecho de proponer guías de trabajo asincrónico benefició también al estudiantado, pues se respetan los distintos ritmos y estilos de aprendizaje. Es decir que el estudiantado puede gestionar su propio aprendizaje bajo la guía de su docente, coincidiendo de esta manera con la investigación de Gao y Zhang (2020) quienes comentan que esta modalidad de enseñanza debería fomentar la autonomía del estudiantado durante su proceso de aprendizaje y la persona docente debería tener un rol de guía y de mediador en lugar de ser un transmisor de conocimientos. Solamente tres docentes hicieron comentarios positivos en cuanto al fenómeno de la virtualidad: "fue interesante, me gustó mucho" (P3, comunicación personal, 07 de junio del 2020), "excelente" (P9, comunicación personal, 06 de julio del 2020), "interesante" (P4, comunicación personal, 07 de junio del 2020), lo que refuerza la idea de que hubo muchas más dificultades que ventajas.

\subsection{Percepciones sobre los cursos que son más aptos para la virtualidad}

En la Tabla 7, se expone que los cursos de la Sección de Francés para otras carreras (impartidos para estudiantes que estudian carreras universitarias de áreas distintas a las lenguas modernas) y los de gramática francesa parecen más aptos para la enseñanza remota. Según estas percepciones, los cursos que son menos aptos para la virtualidad son los de la sección de Francés de Primer año y los de literatura debido a que, según opinan las personas participantes, se necesita una interacción cara a cara entre estudiantes y con la persona docente. 
Pese a esto, hay docentes que tienen percepciones contrarias. Hay quienes consideran que los cursos de francés para otras carreras no son aptos para la virtualidad. Esto lleva a concluir que, el hecho de que un curso sea virtualizable o no, no va a depender precisamente de la persona docente que lo imparta y de sus percepciones, y que esta elección no se debe necesariamente al tipo de curso o a sus objetivos. Los cursos que se eligieron como más aptos para la modalidad virtual fueron aquellos en los que el estudiantado tiene más trabajo autónomo y de preparación de lecturas; por ejemplo, los cursos de gramática, historia y cultura.

En el único curso en el que hay consenso es en el curso de primer año, Francés Integrado I, en el que seis personas lo mencionan como un curso no apto para la virtualidad debido a su naturaleza. En cuanto a los cursos de gramática, cuatro docentes están de acuerdo con que se virtualicen y solamente una persona está en desacuerdo debido al componente oral del curso, por el cual considera necesaria la interacción en modalidad presencial.

Es necesario recordar que esta investigación da cuenta de las percepciones que las personas docentes reportaron durante el I ciclo 2020. En ese momento, hubo docentes que expresaron, por ejemplo, que "aún hay mucho que ajustar" (P12, comunicación personal, 07 de julio del 2020) y que la modalidad virtual "no es lo ideal" (P6, comunicación personal, 06 de julio del 2020). Aunque las percepciones obedecen a una experiencia particular en un modelo de enseñanza remota de emergencia, las percepciones de las personas participantes pueden ser de gran utilidad para los años por venir en el que se puede considerar la virtualidad ya no como una imposición, sino como una elección del estudiantado.

\section{Conclusiones}

La pandemia del COVID-19 llevó al límite las capacidades académicas, personales y tecnológicas del personal docente del mundo entero. En muchos países se tomaron medidas que consistieron cerrar parcialmente los centros educativos al no poder asegurar un relevo de la presencialidad hacia la enseñanza remota de emergencia. En el caso de la UCR, todos los cursos se impartieron de manera virtual y, en el caso del área de francés, fue la primera vez que se propusieron cursos con un componente $100 \%$ virtual.

El I ciclo de 2020 estuvo dominado por paradojas. Por un lado, fue un gran reto académico y personal para la población docente; por otro lado, fue una gran oportunidad para aprender a usar nuevas herramientas tecnológicas, variar y renovar las prácticas metodológicas de las personas docentes. 
Es importante mencionar que, dentro de las ventajas de la virtualidad, las únicas que se citaron fueron relacionadas con el estudiantado: la disponibilidad de material, la autonomía en el aprendizaje. También se mencionó el hecho de no desplazarse al campus como una ventaja innegable tanto para estudiantes como para docentes. Desde el punto de vista académico o personal, ninguna persona docente citó ventajas para el cuerpo docente.

En cuanto a las desventajas de la virtualidad, está claro que las socioafectivas fueron el estrés, el cansancio, el desgaste físico y mental, entre otros. La enseñanza remota a distancia elevó los niveles de estrés en la población docente, lo que provocó un gran deterioro en su estabilidad emocional según la información recabada. Esta situación se puede vincular a dolencias físicas como el dolor de espalda, problemas de insomnio, problemas de visión, etc. A partir de la situación vivida en 2020, se ha evidenciado la importancia de que el personal docente cuente con especialistas a los que pueden acudir en situaciones que atenten contra su salud mental. Es importante mencionar que estos servicios existen en la UCR para el estudiantado y en mucha menor medida para el personal administrativo y docente.

La información recabada lleva a proponer otras conclusiones de orden académico. Con los cursos remotos, el estudiantado es quien está en el centro del aprendizaje. Al haber mayor autonomía y al responsabilizar aún más al estudiantado universitario de su éxito académico individual, la interacción con otras personas estudiantes, los intercambios en el aula y fuera de ella y los procesos de socialización a los que está expuesto el estudiantado, pueden influir negativamente en la constitución de una cultura de grupo, necesaria para el aprendizaje integral y para el desarrollo de competencias generales tal y como se comprenden en el Marco Común Europeo para las Lenguas (MCERL) (2001). Recordemos que también en el grupo se construye el aprendizaje, se refuerza la empatía y muchos otros valores necesarios para el futuro profesional de las personas estudiantes.

Asimismo, se considera que en las respuestas hay elementos que sugieren que el rol de la persona docente ha empezado a transformarse. Al haber siempre un componente asincrónico en los cursos virtuales, la persona docente pasa a ser más un creador de contenido que orienta al estudiante, se ve opacada la figura tradicional de la persona que solo transmite conocimientos.

La población docente estima que la disponibilidad del material de cada curso fue algo muy positivo y que la mayor dificultad constituye la falta de interacción real descrita en párrafos anteriores. Se concluye que también en el ámbito universitario hubo evidencia de la desigualdad en cuanto al acceso a dispositivos informáticos y a Internet. 
Por medio del análisis de las percepciones del personal docente, en este estudio se invita a reflexionar sobre la importancia del papel desempeñado por las personas docentes durante la pandemia, muchas veces en detrimento de su propia salud física y mental y de sus capacidades para el uso de la tecnología. A este respecto, la UNESCO (2020) señala que la crisis sanitaria hizo recordar el rol crucial de las personas educadoras y, que, por lo tanto, los Estados deben velar por su salud y por el bienestar de quienes trabajan en el ámbito educativo.

Muchos afirman que la virtualidad llegó para quedarse en el ámbito educativo. Desde la perspectiva de las personas docentes encuestadas, la virtualidad debe convertirse en una opción debido a las ventajas que puede representar para algunas personas estudiantes y con la premisa de que, de proponerse cursos virtuales, deben ser planificados minuciosamente y con la debida antelación.

Por último, en cuanto a las limitaciones y a los sesgos de esta investigación, por tratarse de un estudio de tipo cualitativo es difícil realizar una generalización de los resultados y, tampoco se pudo realizar una triangulación de los resultados haciendo una comparación con las percepciones del estudiantado de este grupo de docente con el fin de observar si sus percepciones coinciden. Otra limitación, es el hecho de no haber podido seguir a este mismo grupo de docentes durante el segundo semestre para comparar la experiencia vivida en el primer semestre y en el segundo, con el fin de analizar si se ha producido algún tipo de cambios en sus percepciones.

En cualquier panorama que se esboce, las reflexiones y enseñanzas que se derivan de la pandemia COVID-19 apenas han iniciado y es importante que no se tomen a la ligera si se quiere que se implementen cambios que propicien mejoras concretas en los sistemas de enseñanza. Se espera que la experiencia de la enseñanza remota en situación de emergencia suscite una reflexión profunda y debates no solo para la enseñanza de lenguas extranjeras, sino a nivel de la educación superior. En palabras de Sánchez Mendiola et al. (2020), no se debe olvidar que el papel de la persona docente es de guardián de la esperanza de la evolución de la educación.

\section{Referencias}

Alladatin, Judicaël., Gnanguenon, Augustin., Borori, Abel. y Fonton, Appoline. (2020). Pratiques d'enseignement à distance pour la continuité pédagogique dans les universités béninoises en contexte de pandémie de COVID-19: les points de vue des étudiants de l'Université de Parakou. Revue internationale des technologies en pédagogie universitaire, 17(3), 163-177. doi: https://doi.org/10.18162/ritpu-2020-v17n3-16 
Arias Castillo, Carmen Aura. (2006). Enfoques teóricos sobre la percepción que tienen las personas. Horizontes pedagógicos, 8(1), 9-22. Recuperado de https://dialnet.unirioja.es/servlet/articulo?codigo $=4907017$

Dember, William. y Warm, Joel S. (1990). Psicología de la Percepción. Madrid, España: Alianza Editorial.

El Mousadik, Ibtihal. y Aventad, Malika. (2020). Retour d'expérience d'un enseignement àdistance en temps de crise COVID-19: cas d'un public d'adultes allophones. The Journal of Quality in Education, 10(16), 103-121. doi: https://doi.org/10.37870/joqie.v10i16.229

Fortin, Marie-Fabienne. (2010). Fondements et étapes du processus de recherche: méthodes quantitatives et qualitatives. Quebec, Canadá: Chenelière éducation.

Gao, Lori Xingzhen. y Zhang, Lawrence Jun. (2020). Teacher Learning in Difficult Times: Examining Foreign Language Teachers' Cognitions About Online Teaching to Tide Over COVID-19. Frontiers in Psychology, 11, 1-14. doi: https://doi.org/10.3389/fpsyg.2020.549653

Hodges, Charles., Moore, Stephanie., Lockee, Barb., Trust, Torrey. y Bond, Aaron. (2020). The Difference Between Emergency Remote Teaching and Online Learning. EDUCAUSE Review. Recuperado de https://er.educause.edu/articles/2020/3/thedifference-betweenemergency-remote-teaching-and-online-learning

Kikut Valverde, Lorena. (2020). Análisis de resultados de la evaluación de la virtualización de cursos en la UCR ante la pandemia por COVID-19: Perspectiva estudiantil. San José, Costa Rica: UCR.

Lassassi, Moundir., Lounici, Nadjib., Sami, Lylia., Tidjani, Chemseddine. y Benguerna, Mohamed. (2020). Université et enseignants face au COVID19: L'épreuve de l'enseignement à distance en Algérie. Les cahiers du Cread, 36(3), 397-424. Recuperado de https://www.ajol.info/index.php/cread/article/view/202244

Lessard-Hébert, Michelle., Goyette, Gabriel. y Boutin, Gérald. (1996). La recherche qualitative: fondements et pratiques. Montreal, Canadá: Éditions nouvelles.

Lestiyanawati, Rochyano. y Widyantoro, Arif. (2020). Strategies and Problems Faced by Indonesian Teachers in Conducting E-Learning System During COVID-19 Outbreak. Journal of Culture, Literature, Linguistics and English Teaching, 2(1), 71-82. Recuperado de https://ojs.unsiq.ac.id/index.php/cllient/article/view/1271

Lie, Anita., Mina Tamah, Siti., Gozali, Imelda., Retno, Katarina., Diah, Tresiana. y Jemadi, Fransiskus. (2020). Secondary School Language Teachers' Online Learning Engagement during the COVID-19 pandemic in Indonesia. Journal of Information Technology Education: Research, 19, 803-832. doi: https://doi.org/10.28945/4626

Lollia, Manuel. y Issaieva, Elisabeth. (2020). Comment les enseignants assurent la continuité pédagogique et évaluent en contexte de pandémie? Une étude en Guadeloupe. Évaluer. Journal International de recherche en éducation et formation, 1, 181-192. Recuperado de http://journal.admee.org/index.php/ejiref/article/view/231 
Louiz, Driss. (2020). Enseigner à distance via "Google Classroom" au temps de la COVID-19: partage d'une expérience. Revue langues, cultures et sociétés, 6(2), 96-105. doi: https://doi.org/10.48384/IMIST.PRSM/lcs-v6i2.23146

Macintyre, Peter., Gregersen, Tammy. y Mercer, Sarah. (2020). Language teachers 'coping strategies during the COVID-19 conversion to online teaching: Correlations with stress, wellbeing and negative emotions. System, 94, 1-13. doi https://doi.org/10.1016/j.system.2020.102352

Marques Graells, Pere. (2013). Impacto de las TIC en la educación: funciones y limitaciones. 3c TIC: cuadernos de desarrollo a las TIC, 2(1), 1-15. Recuperado de https://dialnet.unirioja.es/servlet/articulo?codigo $=4817326$

Oviedo, Gilberto Leonardo. (2004). La definición del concepto de percepción en psicología base en la teoría Gestalt. Revista de Estudios Sociales, 18, 89-96.

Parot de Sousa, Claire. y Voos Kaspary, Cintia. (2020). La conception d'une discipline de formation d'enseignants dans le contexte de FLE. Revista Letras Raras, 9, 44-62. doi: http://dx.doi.org/10.35572/rlr.v9i5.1991

Prieto Urgilés, Fabiola Isabel., Rodríguez Cajamarca, Lilia Patricia. y Verdugo González, Ruth Concepción. (2020). Fortalezas y debilidades de la pedagogía en el ciberespacio en época de cuarentena COVID-19. Revista Scientific, 5(17), 252-266. doi: https://doi.org/10.29394/Scientific.issn.2542-2987.2020.5.17.13.252-266

Ramos-Huenteo, Valentina., García-Vásquez, Héctor., Olea-González, Constanza., LobosPeña, Karla. y Sáez-Delgado, Fabiola. (2020). Percepción docente respecto al trabajo pedagógico durante la COVID-19. CienciAmérica, 9(2), 1-20. doi: http://dx.doi.org/10.33210/ca.v9i2.325

Rosales Sánchez, Juan. (2015). Percepción y experiencia. Episteme, 35(2), 1-12. Recuperado de http://ve.scielo.org/scielo.php?pid=S0798-43242015000200002\&script=sci arttext

Sánchez García, Adrían. (2020). Microteaching at home: fomento de la creatividad y el aprendizaje "lúcdico" en la Universidad en tiempos de la COVID-19. Magister, 32(1), 6265.

Sánchez Mendiola, Melchor., Martínez Hernández, Ana María., Torres Carrasco, Ruth., de Agüero Servin, Mercedes., Hernández Romo, Alan., Benavides Lara, Mario., Rendón Cazales, Víctor. y Jaimes Vergara, Carlos. (2020). Retos educativos durante la pandemia de COVID-19: una encuesta a profesores de la UNAM. Revista Digital Universitaria, 21(3), 1-24. doi: http://doi.org/10.22201/codeic.16076079e.2020.v21n3.a12

Serrahima, Carlota. (2015). Percepción y conceptos: McDowell y Husserl sobre los contenidos de la experiencia. Investigaciones fenomenológicas, 5, 311-329. Recuperado de https://dialnet.unirioja.es/servlet/articulo?codigo $=7579763$

Silas Casillas, Juan Carlos. y Vázquez Rodríguez, Sylvia. (2020). El docente universitario frente a las tensiones que le plantea la pandemia: Resultados de un estudio mexicano/latinoamericano. Revista Latinoamericana de Estudios Educativos, 50(Especial), $\quad 89-120 . \quad$ Recuperado de https://www.redalyc.org/jatsRepo/270/27063237022/html/index.html 
Tejedor, Santiago., Cervi, Laura., Tusa Fernanda. y Parola, Alberto. (2020). Educación en tiempos de pandemia: reflexiones de alumnos y profesores sobre la enseñanza virtual universitaria en España, Italia y Ecuador. Revista Latina de Comunicación Social, 78, 121. doi: https://www.doi.org/10.4185/RLCS2020-1466

UNESCO. (2020). Note de synthèse: L'éducation en temps de COVID-19 et après. New York, Estados Unidos: ONU.

Vargas Melgarejo, Luz María. (1994). Sobre el concepto de percepción. Alteridades, 4(8), 4753. Recuperado de https://alteridades.izt.uam.mx/index.php/alte/article/view/588

Villanueva Paredes, Grace Ximena., Calcina Málaga, Kimberley Geraldine., Chipa Pandia, Karina Paola., Fuentes Calsina, Alexandra Julia. y Suxso Condori, Jaqueline Diana. (2020). Satisfacción del estudiante respecto a la educación virtual en tiempos de COVID19. Revista Postgrado Scientiarvm, 6(1), 13-17. Recuperado de http://scientiarvm.org/cache/archivos/PDF 013259867.pdf 


\section{Anexo 1}

Cuestionario aplicado al personal docente

Objetivo: Esta encuesta tiene como objetivo conocer su percepción y su experiencia con respecto a la virtualización de los cursos de la carrera en el I-semestre del 2020 durante el contexto de la pandemia COVID-19.

Le tomará 30 minutos para contestar el cuestionario.

\section{Información personal}
1. Sexo:
a. Hombre
b. Mujer

2. Favor marque la opción que corresponda a su rango de edad:
a. 25 a 30 años
b. 31 a 35 años
c. 36 a 40 años
d. 41 o 45 años
e. 46 o 50 años
f. 51 a 55 años
g. 56 o más

3. Favor marque el tiempo de laborar en la Universidad de Costa Rica:
a. 1 a 5 años
b. 6 a 10 años
c. 11 a 15 años
d. 16 a 20 años
e. 21 a 25 años
f. 26 o más

4. Favor marque la opción que corresponda a su carga académica en este semestre:
a. $1 / 8$
b. $2 / 8$
c. $3 / 8$
d. $4 / 8$
e. $5 / 8$
f. $6 / 8$
g. $7 / 8$
h. $8 / 8$

5. Actualmente, usted está en:
a. propiedad

b. interino

6. Favor marque la opción que corresponda a su último título obtenido:
a. Bachillerato
b. Licenciatura
c. Maestría
d. Doctorado 


\section{Información sobre la experiencia de impartir o recibir cursos en modalidad virtual o bimodal}

1. ¿Ha tenido experiencias previas impartiendo cursos de manera bimodal?:

a. Sí

b. No (pasar a la pregunta 3)

2. Si su respuesta anterior fue afirmativa, indicar en dónde ha tenido experiencia impartiendo cursos de manera bimodal:

3. ¿Ha tenido experiencias previas impartiendo cursos de manera virtual?:

a. Sí

b. No (pasar a la pregunta 5)

4. Si su respuesta anterior fue afirmativa, indicar en dónde ha tenido experiencia impartiendo cursos de manera virtual:

5. ¿Ha recibido cursos de manera virtual?:

a. Sí

b. No (pasar a la pregunta 7)

6. Si su respuesta anterior fue afirmativa, indicar en dónde ha recibido cursos de manera virtual:

7. ¿Ha recibido cursos de manera bimodal?:

a. Sí

b. No (pasar a la pregunta 9)

8. Si su respuesta anterior fue afirmativa, indicar en dónde ha recibido cursos de manera bimodal:

9. ¿Ha usado la plataforma de Mediación Virtual anteriormente?:

a. Sí

b. No

10. ¿Para sus cursos, en el I-semestre, está usando la plataforma de Mediación Virtual?

a. Sí

b. No (pasar a la pregunta 12)

11. ¿Qué le ha parecido la plataforma? 
12. ¿Cuáles herramientas ha empleado para sus cursos en la plataforma de Mediación Virtual? (puede marcar varias opciones)
a. foro
b. wiki
c. envío de tareas o trabajos
d. examen en línea
e. depósito de materiales para el curso
f. juegos en línea
g. otro:

13. ¿Cuáles recursos ha utilizado para crear su contenido pedagógico? (puede marcar varias opciones)
a. Drive
b. Creación de audios
c. Creación de videos
d. Quizlet
e. Kahoot
f. Google classroom
g. Vocaroo
h. Screencast-O-matic
i. Youtube
j. Powtoon
k. Padlet
I. WhatsApp
m. Canvas
n. Powerpoint
o. otros:

14. ¿Qué herramientas le gustaría aprender a usar?

\section{Percepción sobre la experiencia de impartir cursos en el contexto de la pandemia}

1. ¿Cómo ha sido su experiencia al haber impartido los cursos de la carrera en el contexto de la pandemia del COVID-19?

2. ¿Indicar cuál fue la cantidad de estudiantes matriculados en su curso o sus cursos?

3. ¿Cuáles son las ventajas de impartir cursos en su contexto de la pandemia según su experiencia en este semestre?

4. ¿Cuáles son las dificultades de impartir cursos en su contexto de la pandemia según su experiencia en este semestre? 
5. ¿Cuáles son los inconvenientes de impartir cursos en su contexto de la pandemia según su experiencia en este semestre?

6. De los cursos que impartió este semestre, ¿cuáles considera que son más aptos para la virtualidad? Explicar

7. De los cursos que impartió este semestre, ¿cuáles considera que son menos aptos para la virtualidad? Explicar

8. De los cursos que impartió, ¿cuáles considera que podrían seguir ofreciéndose en la modalidad virtual? Explicar

9. ¿Cuántos estudiantes debería tener un curso en la modalidad virtual?

10. ¿Cómo se sintió al impartir los cursos durante el contexto de la pandemia?

11. ¿Qué tipo de capacitaciones necesitaría para seguir impartiendo cursos en el contexto de la pandemia?

12. ¿Qué tipo de herramientas de TIC necesitaría que lo capacite para seguir impartiendo cursos en el contexto de la pandemia?

13. ¿Cuáles temas le gustaría que se trataran en las capacitaciones?

14. Comentarios: 
Revista indizada en

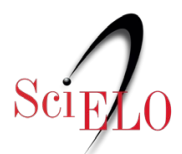
redalyc latindex

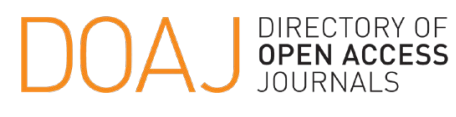

Distribuida en las bases de datos:

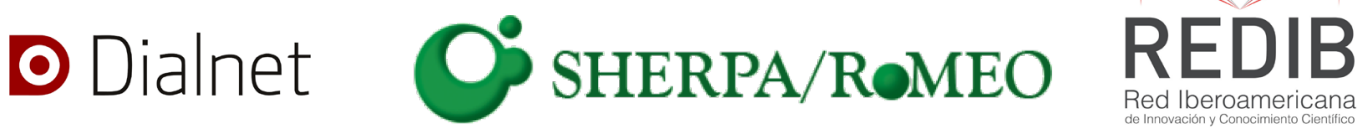

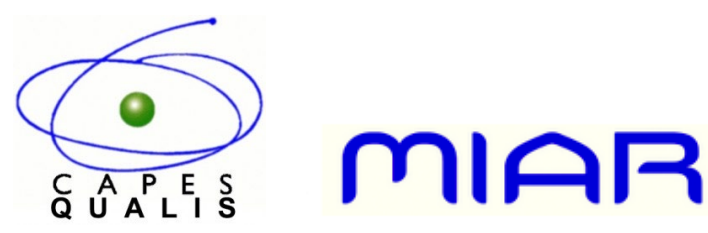

